\title{
ppohDEM: Computational Performance for Open Source Code of the Discrete Element Method
}

\author{
Daisuke Nishiura, $^{\mathrm{a}, *}$, Miki Y. Matsuo ${ }^{\mathrm{a}}$, Hide Sakaguchi ${ }^{\mathrm{a}}$ \\ ${ }^{a}$ Institute for Research on Earth Evolution, Japan Agency for Marine-Earth Science and \\ Technology, Kanagawa 236-0001, Japan
}

\begin{abstract}
We investigate performance improvements for the discrete element method (DEM) used in ppohDEM. First, we use OpenMP and MPI to parallelize DEM for efficient operation on many types of memory, including shared memory, and at any scale, from small PC clusters to supercomputers. We also describe a new algorithm for the descending storage method (DSM) based on a sort technique that makes creation of contact candidate pair lists more efficient. Finally, we measure the performance of ppohDEM using the proposed improvements, and confirm that computational time is significantly reduced. We also show that the parallel performance of ppohDEM can be improved by reducing the number of OpenMP threads per MPI process.
\end{abstract}

Keywords: open source, discrete element method, particle, parallelization, high performance computing

\section{PROGRAM SUMMARY}

Manuscript Title: ppohDEM: Computational Performance for Open Source Code of the Discrete Element Method

Authors: Daisuke Nishiura, Miki Yamamoto, Hide Sakaguchi

Program Title: ppohDEM

Journal Reference:

Catalogue identifier:

Licensing provisions: none

Programming language: Fortran

Computer: CPU based workstations and parallel computers

\footnotetext{
${ }^{*}$ Corresponding author.

E-mail address: nishiura@jamstec.go.jp

Preprint submitted to Computer Physics Communications December 19, 2013
}

(C) 2014. This manuscript version is made available under the Elsevier user license

http://www.elsevier.com/open-access/userlicense/1.0/ 
Operating system: Linux, Windows

$R A M$ : Dependent upon the numbers of particles and contact particle pairs (1GB for the example program supplied with the package)

Number of processors used: Tested with up to 8 processors

Keywords: DEM, Particle, Collision, Parallel computing

Classification: 6.5 Software including Parallel Algorithms, 13 Geophysics and Atmospheric Science

External routines/libraries: MPI-2, OpenMP

Nature of problem:

Collision dynamics of viscoelastic particles with friction in powder engineering and soil mechanics.

Solution method:

Parallelized DEM running on shared and/or distributed systems is the solution method based particle model in which geometrical size and shape attributes are provided for each element. In the DEM, the Voigt model and Coulomb friction model are considered at each contact point between particles.

Running time:

10 minutes for the example program supplied with the package using $2 \mathrm{CPU}$ of Intel Xeon E7-4870

\section{Introduction}

Scientists and engineers often hesitate to use numerical simulation even when they intend to apply numerical methods to their research. There are a number of reasons for this hesitation: program code can be very complex, computational costs can be high, and efficient parallelization can impose significantly greater complexity. To address these problems, an open source infrastructure for implementing and executing optimized and reliable simulations on parallel computers has been developed [1-3]. Known as ppOpenHPC [4], this infrastructure consists of several libraries covering a variety of scientific computations, such as the finite difference method (FDM), the finite element method (FEM), the finite volume method (FVM), the boundary element method (BEM), and the discrete element method (DEM). In this paper, we focus on improving the computational performance of ordinary DEM in support of ppohDEM, the open source version of DEM [5].

DEM is a popular method for simulating the contact dynamics of particles, such as powders and grains, for which geometrical size and shape attributes are provided for each body. DEM is a so-called "soft core model" 
allowing a degree of overlap when two elements are in contact. In the most conventional formulation of DEM, the Voigt model is applied at each contact point in both the normal and tangential directions. In the tangential direction, Coulomb friction is introduced to determine the maximum tangential force and the slip condition. At the moment, DEM is widely used to simulate granular materials such as sand, pebbles, and other grains [6-11] in applications involving powder technology and geotechnical engineering. Notable open source DEM frameworks developed for these domains include LAMMPS [12], ESyS-Particle [13], YADE-OPEN DEM [14], and OpenFOAM [15].

Unfortunately, the number of particles and time steps involved in DEM, and the resulting computational costs are often too large to handle on current personal computers or workstations, so practical DEM simulations are usually performed on supercomputers or PC clusters. To support effective use of these computing resources, a number of parallel DEM algorithms for shared memory have been developed $[16,17]$. Some of these parallel algorithms [16] are integrated into a commercial DEM software package called "DEMIGLACE" [18] from JAMSTEC. Several parallel algorithms for use with distributed memory have also been developed [19-24], emphasizing dynamic load balancing [25, 26] for efficient execution of DEM on massive, distributed parallel computing systems. Unfortunately, when using these systems, computational improvement begins to degrade in excess of several dozens of CPUs, as inter-process communication becomes too burdensome. To improve the distributed parallel performance of DEM, we need to investigate not only dynamic load balancing but also possible reductions in the number and volume of inter-process communications, as well as the serialization of the memory address where communicated data for discrete particles is stored. We also need to create a contact candidate pair list to reduce the frequency and scope of searches for neighbor particles, while recognizing that the computational cost of creating this list is especially large for DEM operations [27].

To address these issues, we propose a new open source DEM framework called "ppohDEM," based on the following design priorities:

(1) To make optimized and parallelized DEM code easy to read, modify, and apply, even for novice programmers, ppohDEM is written entirely in basic Fortran, thus avoiding the conceptual complexities of higher-level programming languages.

(2) ppohDEM implements a simple and efficient inter-process communica- 
tion algorithm for 1-D domain decomposition.

(3) ppohDEM provides a prototype of DEM code for coupling with other libraries within the ppOpen-HPC framework [4].

In this paper, we begin by describing our new algorithm for the descending storage method (DSM), which uses the sorted order of particle labels to speed creation of contact candidate pairs. Next, we reduce the complexity and data volume of inter-process communications by using sorted particle labels and applying action-reaction law to the summation of inter-particle contact force. Finally, we measure the computational performance of the improved DEM code to find an optimal parallel task balance between the number of OpenMP threads on shared memory and the number of MPI processes on distributed memory.

\section{Overview of Computational Procedure in ppohDEM}

An overview of the computational flow in ppohDEM is provided in Fig. 1. First, initial parameters, such as the size of the computational system and the material properties of the simulated particles, are set and some number of particles are created and located in the system. Next, the list of contact candidate pairs is created in order to efficiently detect particle pairs in contact. Contact between particles in the list is then detected and the contact force is calculated. The contact force is distributed in accordance with the action-reaction law and summed up for each particle. Finally, the velocities and positions of particles are updated by integrating Newton's motion equation for the contact force. These steps are iterated in constant discrete time until the simulation reaches its intended result. During iteration, the list of contact candidate pairs is updated in according to a set frequency. The details of the major steps (i.e. creation of the contact candidate list, calculation of contact force, and integration of motion equation) are explained below.

\subsection{Creating the List of Contact Candidate Pairs}

To create the list of contact candidate pairs efficiently, ppohDEM uses the descending storage method (DSM). Cubic cells with an edge length of $D_{\mathrm{p}}(1.0+\alpha)$ are arrayed within the computational region, as shown in Fig. 2, so that every particle can be assigned to a single cell based on its location. Here, $D_{\mathrm{p}}$ is the particle diameter, and $\alpha$ is a constant parameter for adjusting the update frequency of the list of contact candidate pairs. First, a particle 
label is stored in the array Norderp as cell data that corresponds to the position of each particle, as seen in Code 1 . Here, $N_{\mathrm{p}}$ is the total number of particles in the system, Numcl is the number of particles in each cell, and $i b$ is the array of cell labels corresponding to the positions of all particles. The value at $\operatorname{Norderp}\left[1,1: N_{\text {cell }}\right]$ is set to 1 during initialization. In DSM, particle labels are stored to Norderp in accordance with the descending order of particle labels each time the contact candidate pair list is updated.

Next, for each particle $i$, the cell to which $i$ belongs, as well as its adjacent cells, are searched for particles with a particle label $j>i$, as seen in Code 2. Here, $I_{\text {list }}$ is the number of contact candidate pairs and $i c$ is the cell label adjacent to $i b_{i}$. This search is relatively efficient because an if statement is 
Figure 2: 1-D domain decomposition in parallel computation on distributed memory using sorted particle labels.

not needed to judge whether $j>i$ and the number of searched adjacent cells is reduced from 27 to 14, as shown by the gray cells in Fig. 3. Line 1 of Code 1 sorts particle labels by cell label, as illustrated in Fig. 2, and cell data is reduced by line 5 in Code 2. For example, when a particle of No. 5 in Fig. 2 searches the adjacent gray cells, particles from No. 1 to No. 4 are invisible for the particle, as shown in Fig. 4(a). In addition, the particle cannot look up itself because cell data corresponding to the particle from No. 1 to No. 5 has been erased at line 5 in Code 2. Similarly, when a particle of No. 6 searches adjacent cells as shown in Fig. 4(b), particles of No. 1-5 are erased from the cell data by the time the search for contact candidate pair particles is finished (see Fig. 4(a)).

If the distance between particle $i$ and $j$ is less than $\left(r_{i}+r_{j}\right)(1.0+\alpha)$, where $r_{i}$ represents the radius of the particle $i$, then $i$ and $j$ are added to the contact candidate pair list $L_{\mathrm{p}}$ at line 13 in Code 2 . Contact detection is then performed only for the particle pairs recorded in this candidate pair list. In the corresponding code, the created contact candidate pair list is updated only when the cumulative displacement of a particle has exceeded $r_{i} \alpha$. The infrequency of such updates is crucial to reducing computational cost.

For comparison between an ordinary DEM and ppohDEM, we prepared 


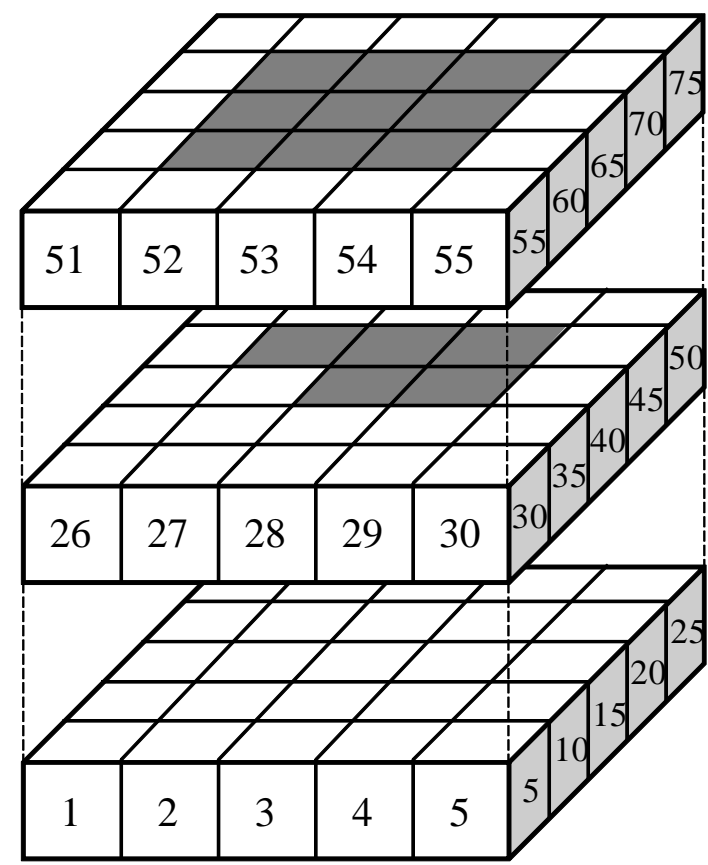

Figure 3: Search region of neighbor particles on 1-D labeling cells.

three fundamental codes: OpenDEM_A, OpenDEM_B, and OpenDEM_C. Corresponding DEM codes are provided in the Appendix A. OpenDEM_A or OpenDEM_B commonly uses the same contact candidate pair list creation method used in a fundamental DEM code, whereas OpenDEM_C only uses a descending storage method (DSM). ppohDEM is represents an improvement over OpenDEM_C, implementing a sort of particle labels by cell labels. This improvement has three benefits:

(1) Reducing the number of searching cells from 27 to 14

(2) Avoiding an if statement involving the magnitude relationship of particle labels

(3) Reducing the Norderp cell data initialization operation for all cell labels at each update of the contact candidate pair list

\subsection{Model and Calculation of Contact Force}

The normal and tangential components $\left(\mathbf{F}_{\mathrm{n}}\right.$ and $\mathbf{F}_{\mathrm{t}}$, respectively) of the contact forces between particles are represented in the Voigt model using a 
(a) Particle of No. 5 searches pair particle after particles of No. 1-4 searched

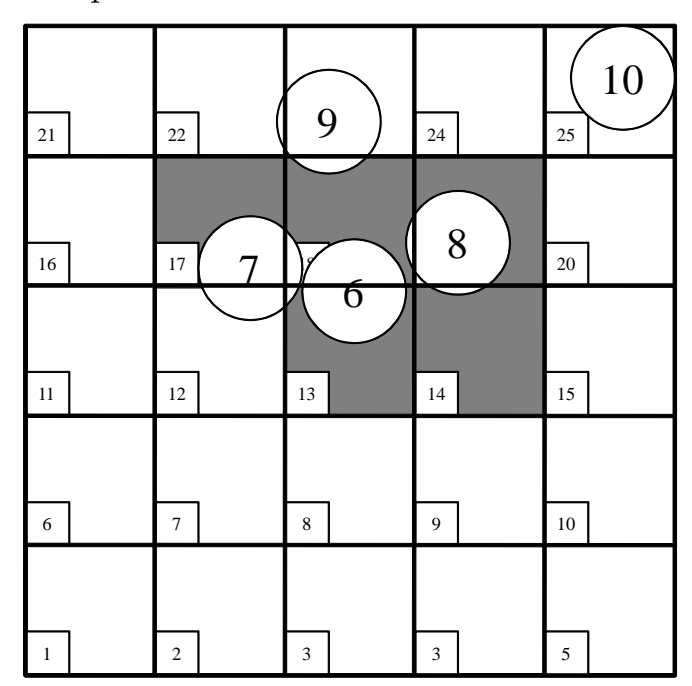

(b) Particle of No. 6 searches pair particle

after particle of No. 5 searched

Figure 4: Pairing process of contact candidate particles by using a DSM with sorted particle label.

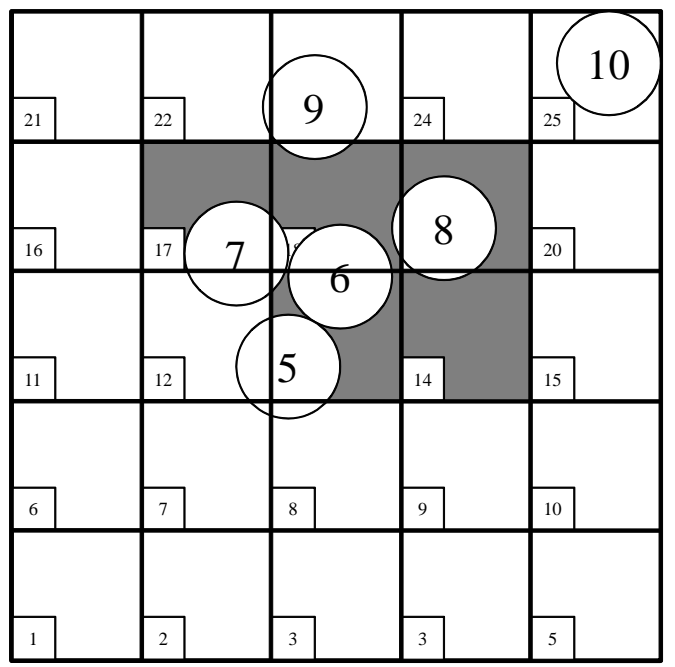




\begin{tabular}{lc}
\hline Code 1 ppohDEM: Sub_Cell \\
\hline $1: \quad$ Call Quicksort \{particle label is sorted by cell label $\}$ \\
$2: \quad$ for $i=N_{\mathrm{p}}$ to 1 do $\{$ particle label is stored as cell data \\
$\quad$ in "descending" order\} \\
3: $\quad i b_{i} \Leftarrow i b[i]$ \\
$4: \quad \quad$ Numcl $\Leftarrow N$ orderp $\left[1, i b_{i}\right]+1$ \\
$5: \quad \quad$ orderp $\left[1, i b_{i}\right] \Leftarrow N u m c l$ \\
$6: \quad \quad N o r d e r p\left[N u m c l, i b_{i}\right] \Leftarrow i$ \\
$7: \quad$ end do \\
\hline
\end{tabular}

frictional slider, as given in (1) and (2) below.

$$
\begin{aligned}
& \mathbf{F}_{\mathrm{n}}=K_{\mathrm{n}} \mathbf{X}_{\mathrm{n}}+\eta_{\mathrm{n}} \mathbf{V}_{\mathrm{n}} \\
& \mathbf{F}_{\mathrm{t}}=\min \left[\mu \mathbf{F}_{\mathrm{n}} \cdot \mathbf{V}_{\mathrm{t}} /\left|\mathbf{V}_{\mathrm{t}}\right|, K_{\mathrm{t}} \mathbf{X}_{\mathrm{t}}+\eta_{\mathrm{t}} \mathbf{V}_{\mathrm{t}}\right]
\end{aligned}
$$

Here, $K$ is the spring constant, $\eta$ is the dumping coefficient, $\mu$ is the Coulomb friction coefficient, $\min []$ is the minimum function, and $\mathbf{X}$ and $\mathbf{V}$ are vectors of relative displacement and relative velocity at the contact point between contact particle pairs, respectively. The translational component $\mathbf{F}=\mathbf{F}_{\mathrm{n}}+\mathbf{F}_{\mathrm{t}}$ and the rotational component $\mathbf{M}=\mathbf{R}_{i} \times \mathbf{F}_{\mathrm{t}}$ of the contact force exerted on particle $i$ by particle $j$ are computed in Code 3 . Here, $\mathbf{R}$ is the position vector that points from the center of a particle to the contact point. Furthermore, the translational force $\mathbf{- F}$ and the rotational force $\mathbf{M}$ are exerted on particle $j$ by particle $i$ in accordance with the action-reaction law, as given at lines 10 and 11 in Code 3, respectively.

\subsection{Integration of Particle Motion Equation}

The equations for translational motion and rotational motion about the center of mass are given by

$$
\begin{aligned}
& m_{\mathrm{p}} \frac{\partial \mathbf{u}_{p}}{\partial t}=\sum \mathbf{F} \\
& I_{\mathrm{p}} \frac{\partial \mathbf{w}_{\mathrm{p}}}{\partial t}=\sum \mathbf{M}
\end{aligned}
$$

where $\mathbf{u}_{\mathrm{p}}$ and $\mathbf{w}_{\mathrm{p}}$ are the particle translational velocity and rotational velocity, and $m_{\mathrm{p}}$ and $I_{\mathrm{p}}$ are the particle mass and moment of inertia, respectively. These motion equations integrated using a simple leapfrog method. 


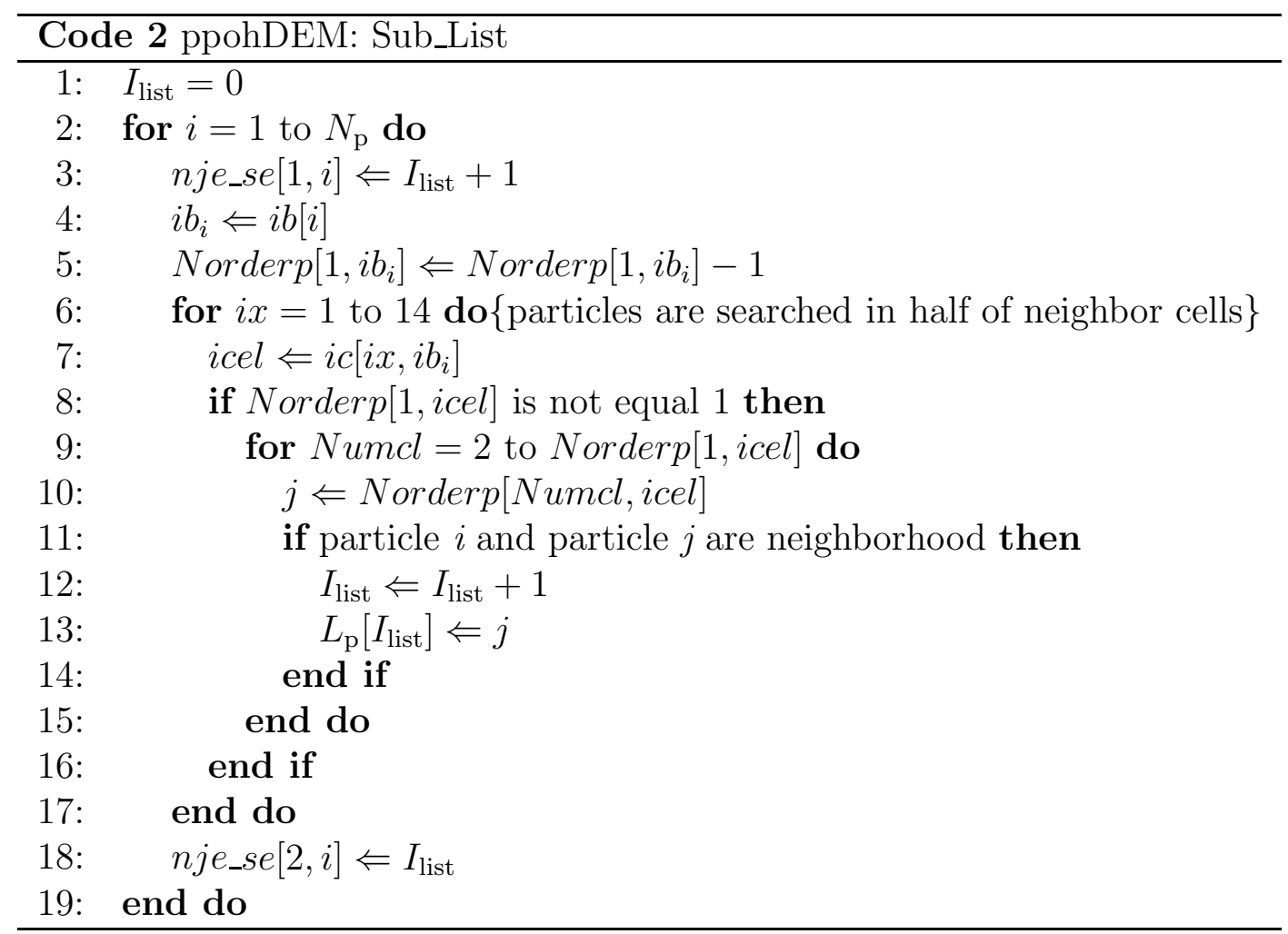

\section{Parallel Computing Method}

In ppohDEM, hybrid parallel computing is enabled on shared memory and distributed memory systems. The shared and distributed parallel computing methods are described in the following subsections.

\subsection{OpenMP Parallelization on Shared Memory}

Parallelization on shared memory is implemented using OpenMP. While this makes it easy to parallelize integration of the motion equation, it does little to help parallelize the creation of the list of contact candidate pairs or the summation of contact force, as these create conflicts in write access to memory. For example, when the list of contact candidate pairs is created, the counting processes for the number of particles in the cell (line 4 in Code 1 ) and the number of lists (line 12 in Code 2) make parallelization impossible. These processes can be parallelized if algorithms described in our past work [16] are used, but these parallel algorithms cannot be freely distributed in accordance with Japanese patent and commercial software licensing. Thus, 


\begin{tabular}{cc}
\hline \multicolumn{2}{c}{ Code 3 ppohDEM: Sub_Actf } \\
\hline $1:$ & for $i=1$ to $N_{\mathrm{p}}$ do \\
$2:$ & $j i k_{\mathrm{s}} \Leftarrow n j e_{-} s e[1, i]$ \\
$3:$ & $j i k_{\mathrm{e}} \Leftarrow n j e_{\text {_se }}[2, i]$ \\
$4:$ & for $j i k=j i k_{\mathrm{s}}$ to $j i k_{\mathrm{e}}$ do \\
$5:$ & $j \Leftarrow L_{\mathrm{p}}[j i k]$ \\
$6:$ & if particle $i$ and particle $j$ are in contact then \\
$7:$ & F and $M$ are calculated by Eqs. $(1)$ and $(2)$ \\
$8:$ & Force $[i] \Leftarrow$ Force $[i]+F$ \\
$9:$ & Torque $[i] \Leftarrow$ Torque $[i]+M$ \\
$10:$ & Force $[j] \Leftarrow$ Force $[j]-F$ \\
$11:$ & Torque $[j] \Leftarrow$ Torque $[j]+M$ \\
$12:$ & end if \\
$13:$ & end do \\
$14:$ & end do \\
\hline
\end{tabular}

in ppohDEM, the summation of contact force in Code 3 is parallelized using the OpenMP directive "!OMP ATOMIC" in lines 10-11.

\subsection{MPI Parallelization on Distributed Memory}

The total computational region is split along the $\mathrm{Z}$-axis into a number of sub regions equal to the number of MPI processes. One MPI process communicates with neighbor processes, and the width of the communication region is the size of one cell in the Z-axis direction. Each MPI process creates a list of contact candidate pairs, calculates the forces, and updates the position and velocity of particles within the associated region. When the list of contact candidate pairs is updated, particle labels are sorted by cell labels in each MPI process region, as shown in Fig. 2. This sort technique reduces the cost of MPI communication because particle and contact force data can be sent and received using sequential memory addresses. For example, in Fig. 2, MPI communication can send particle data using the sequential address from MPI process No. 0 to MPI process No. 1 because the particle labels from No. 3 to No. 6 are sequential, due to sorting. Moreover, one-side communication is sufficient for computing the inter-particle contact force and to create the list of contact candidate pairs, since the search region of contacted particles is restricted to the upper part of the cell with the given particle along the Zaxis, as shown in Fig. 3. Then, if the inter-particle contact force is computed 
in the region of the MPI process for No. 0, as shown in Fig. 2, the MPI process for No. 1 sends the data of particles of No. 7 and No. 8, and the process for No. 0 simply receives the data. The contact force of particle pair across processes is computed in the MPI process for No. 0, i.e., the contact force exerted on particles of No 7 and No. 8, must be sent to the process for No. 1. These procedures in terms of MPI communication are summarized in Code 4 , where $N_{\text {lo }}$ is the number of particles in the region where data is received from lower side process, and $F_{\text {lo }}$ and $M_{\text {lo }}$ are force and torque received from the lower side process, respectively. Note that particles are globally labeled through all MPI processes in Fig. 2 but particles are locally labeled by each MPI process in Code 4.

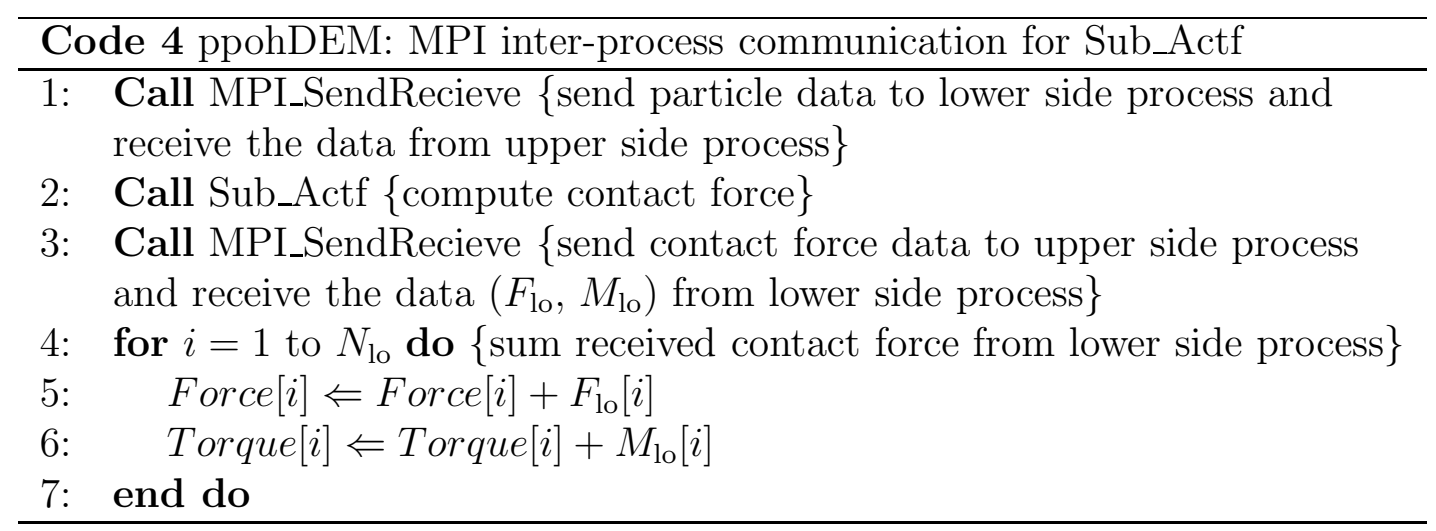

\section{Performance Evaluation}

The computational system size and the number of particles used in our test program are provided in Table 1. Particles are initially placed using a simple cubic lattice in the system, where the lattice size is equal to the particle diameter. Our performance test of ppohDEM is run under static conditions without gravity, so particles need not be moved during the computation. To measure the computational time elapsed for calculating contact force in Sub_Actf, calculation is performed for all contact candidate pairs by ignoring the if statement at line 6 in Code 3. Moreover, the creation of contact candidate pairs is done for every time step and elapsed time is measured. The computation is run over 10,000 time steps to obtain the average computational time per step. Details of the workstation used for the test are given in Table 2 . 
Table 1: System size and the number of particles used in computational performance test of DEM, where the system is of cubic shape and $W$ is the length of one side of the cubic system divided by the particle diameter

\begin{tabular}{lcccccc}
\hline Test case No. & 1 & 2 & 3 & 4 & 5 & 6 \\
\hline $\begin{array}{l}\text { System size divided by } \\
\text { particle diameter }(W)\end{array}$ & 8 & 16 & 32 & 64 & 128 & 256 \\
Number of particles & 512 & 4,096 & 32,768 & 262,144 & $2,097,152$ & $16,777,216$ \\
\hline
\end{tabular}

Table 2: Profile of test platform used in DEM simulations

\begin{tabular}{lll}
\hline OS & CentOS 5.8 x64 \\
Number of CPUs in a system & 4 & \\
\hline \multicolumn{3}{c}{ CPU } \\
\hline Processor name & Intel Xeon E7-4870 \\
Frequency of processor & 2.4 & {$[\mathrm{GHz}]$} \\
Number of cores per processor & 10 & {$[-]$} \\
Processor peak performance & 96 & {$[\mathrm{Gflops}]$} \\
L1 cache & 32 & {$[\mathrm{~KB}]$} \\
L2 cache & 256 & {$[\mathrm{~KB}]$} \\
L3 cache & 30 & {$[\mathrm{MB}]$} \\
\hline \multicolumn{4}{c}{} \\
\hline Frequency & 1066 & {$[\mathrm{MHz}]$} \\
Granularity & 512 & {$[\mathrm{~GB}]$} \\
\hline
\end{tabular}

\subsection{Computational Performance of DEM on a single CPU}

To confirm improvements in computational performance, we measured elapsed times for ppohDEM code and fundamental DEM codes (i.e. OpenDEM_A, OpenDEM_B, and OpenDEM_C) for case No. 5 in Table 1, using a single CPU parallelized by 10 threads in OpenMP. We also measured the computational time for DEMIGLACE and compared it to that of ppohDEM. Average elapsed times for subroutines Sub_Cell, Sub_List, and Sub_Actf, corresponding to Codes 1, 2 and 3, respectively, are shown in Fig. 5. To clarify the differences between fundamental DEM codes, DEMIGLACE, and ppohDEM, the salient design features of these codes, and their raw performance data (as visualized in Fig. 5), are summarized in Table 3.

For the Sub_Cell subroutine, in which particle labels are stored in cell data, OpenDEM_B performs the same as OpenDEM_A because these codes are same. The code of OpenDEM_C, unfortunately, does not seem to be 
Table 3: Summary of algorithms and computational performances for each DEM code

\begin{tabular}{l|l|l|c|c|c|c}
\hline \multirow{2}{*}{ Code name } & \multicolumn{1}{|c|}{ Salient design features } & \multicolumn{3}{c}{ Elapsed time per step [ms] } \\
\cline { 2 - 7 } & $\begin{array}{l}\text { Contact force summation } \\
\text { parallelization method. }\end{array}$ & $\begin{array}{l}\text { Contact candidate pair list } \\
\text { creation method. }\end{array}$ & Sub_Actf & Sub_Cell & Sub_List & Total \\
\hline OpenDEM_A & $\begin{array}{l}\text { Double calculation } \\
\text { without action-reaction } \\
\text { law }\end{array}$ & $\begin{array}{l}\text { No DSM. List contains all } \\
\text { neighbor particles } j \text { with } i>j \\
\text { and } i<j .\end{array}$ & 0.0498 & 10.5270 & 112.9602 & 123.5370 \\
\hline \multirow{2}{*}{ OpenDEM_B } & $\begin{array}{l}\text { OpenMP atomic-add } \\
\text { instruction. }\end{array}$ & $\begin{array}{l}\text { No DSM. List contains only } \\
\text { neighbor particles } j \text { with } i<j .\end{array}$ & 0.0501 & 10.8070 & 65.7731 & 76.6302 \\
\hline ppohDEM & $\begin{array}{l}\text { OpenMP atomic-add } \\
\text { instruction }\end{array}$ & $\begin{array}{l}\text { DSM-based. List contains only } \\
\text { neighbor particles } j \text { with } i<j . \\
\text { (The cost of initialization for } \\
\text { cell data is reduced by DSM.) }\end{array}$ & 0.0502 & 11.2317 & 58.7864 & 70.0683 \\
\hline instruction & $\begin{array}{l}\text { Sort technique is applied to } \\
\text { OpenDEM_C. Cell search area } \\
\text { is half that of other codes. }\end{array}$ & 0.0505 & 10.1421 & 51.2113 & 61.4039 \\
\hline
\end{tabular}

an improvement over that of OpenDEM_B, even though the initialization operation of cell data is reduced by using the DSM. Moreover, ppohDEM, which adds a sorting operation to the code of OpenDEM_C, is slightly faster than OpenDEM_C (sorting costs included) due to an improvement in the cache hit ratio of sorted data. For Sub_List, OpenDEM_B is $47 \mathrm{~ms}$ (42 $\%$ ) faster than OpenDEM_A, since the number of contact candidate pairs is halved. OpenDEM_C is $7.0 \mathrm{~ms}(11 \%)$ faster than OpenDEM_B because the conditional branching of the if statement for the magnitude of particle labels is avoided by using the DSM. ppohDEM is $7.6 \mathrm{~ms}(13 \%)$ faster than OpenDEM_C because the number of searched cells is halved when using sorted particle labels. As a result of these improvements, creation of the list of contact candidate pairs in ppohDEM is $62 \mathrm{~ms}(50 \%)$ faster than in OpenDEM_A.

For subroutine Sub_Actf, in which contact force is calculated, OpenDEM_A is slightly faster than the others, even though the number of contact candidate pairs in OpenDEM_A is doubled. This is because OpenDEM_A can be fully parallelized, whereas others must use an OpenMP synchronization directive. However, in comparison with ppohDEM, the computational time of Sub_Actf is only $0.7 \mu$ s lower, a negligible amount compared to the $62 \mathrm{~ms}$ improvements for Sub_Cell and Sub_List. Thus, we conclude that ppohDEM is the most computationally efficient when considering the entirety of the source code. 


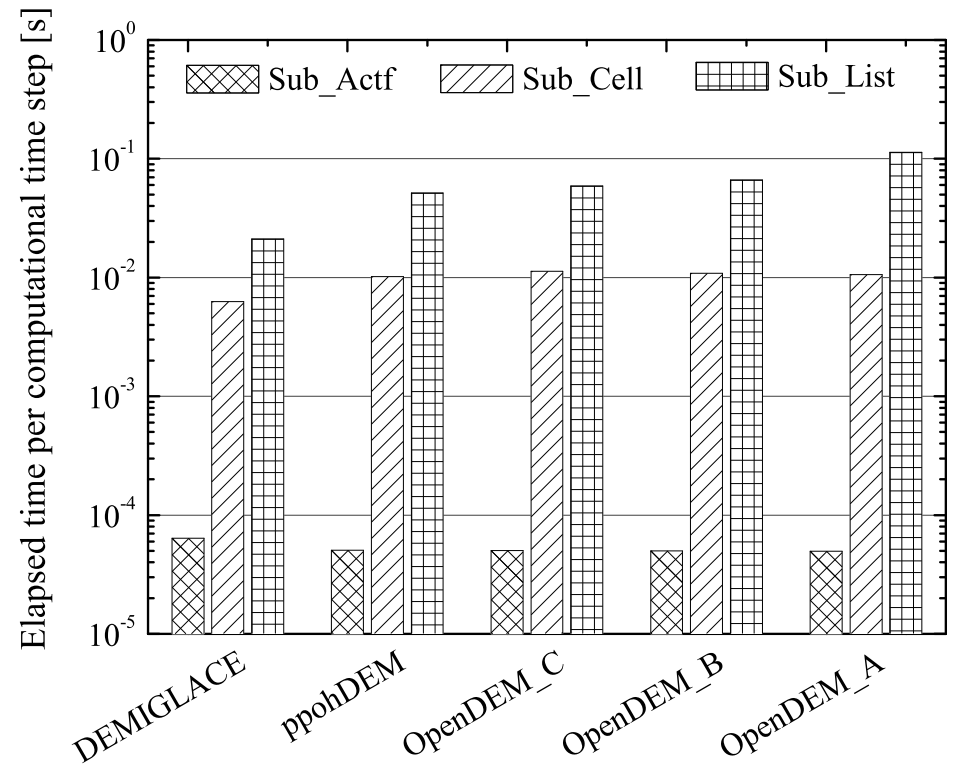

Figure 5: Elapsed time for each DEM simulation using different algorithms.

The performance of DEMIGLACE was found to be more efficient than ppohDEM for the subroutines of contact candidate pair creation, since DEMIGLACE is fully parallelized on shared memory. Even so, Sub_Actf in DEMIGLACE was slower than the others due to DEMIGLACE's inefficient memory access when summing the contact force (using a reference table to avoid synchronization between OpenMP threads). In addition, by allocating the array of the reference table in physical memory, the memory usage in DEMIGLACE nearly doubles that in ppohDEM (see Table 4). The memory usage in ppohDEM is the lowest of all because the size of the lists of contact candidate pairs is half that of OpenDEM_A. Moreover, the memory usage for the buffer array of data used in packing/unpacking for MPI communications is unnecessary in ppohDEM, as mentioned in Section 4.3.

As a result, we can confirm that ppohDEM improves computational performance and memory usage compared to fundamental DEM code.

\subsection{Parallel Performance on Shared Memory}

We measured the parallel performance on shared memory under the conditions in Table 1. The relationship between computational time and the 
Table 4: Physical memory usage on each DEM code

\begin{tabular}{c|c|c|c|c|c}
\hline & \multirow{2}{*}{ DEMIGLACE } & \multirow{2}{*}{ ppohDEM } & \multicolumn{3}{|c}{ OpenDEM } \\
\cline { 4 - 6 } & & & $\mathrm{A}$ & $\mathrm{B}$ & $\mathrm{C}$ \\
\hline $\begin{array}{c}\text { Used physical } \\
\text { memory [GB] }\end{array}$ & 11 & 6.8 & 7.2 & 6.9 & 6.9 \\
\hline
\end{tabular}

number of CPU cores used for ppohDEM and DEMIGLACE is shown in Figs. 6 and 7, respectively. To further clarify scaling performance, the data used in Figs. 6 and 7 is also provided in raw form in Tables B.1 and B.2, respectively, of Appendix B. Here, the source code used in the measurement is not parallelized on distributed memory by MPI. Note that the parallel performances of ppohDEM and DEMIGLACE are poor when the number of particles is small, due to the overhead associated with parallelization. The elapsed time for the creation of contact candidate pairs in ppohDEM is shown in Fig. 6(a), where the computational time is constant against the number of used CPU cores. This did not improve even when the number of particles was increased. The elapsed time for the calculation of contact force in ppohDEM is shown in Fig. 6(b). Note that the computational time seems to decrease when the number of CPU cores is increased from 10 to 30, but increases at $40 \mathrm{CPU}$ cores. This is likely due to the synchronous operation needed for the summation of contact force, and because the effect of data locality was increased due to the cache is not shared between CPUs. On the other hand, parallel performances of contact force calculation and contact candidate pair creation in DEMIGLACE (see Fig. 7) are nearly good enough to use many cores (up to 30) on shared memory, except when the number of particles is small. The performance also seems to be best when the number of particles is 262,144 of case No. 4 in Table 1. We conclude that in its current form, ppohDEM is best when used on computer systems with relatively few CPU cores because the parallel performance is not enough to create contact candidate pairs, although the performance of contact force calculation is even equal or better than that of commercial software as DEMIGLACE.

\subsection{Parallel Performance on Distributed Memory}

We also measured parallel performance on distributed memory. The effect of the number of MPI processes on computational time is shown in Fig. 8. Our test focused on the case of No. 5 in Table 1. We performed a strong 


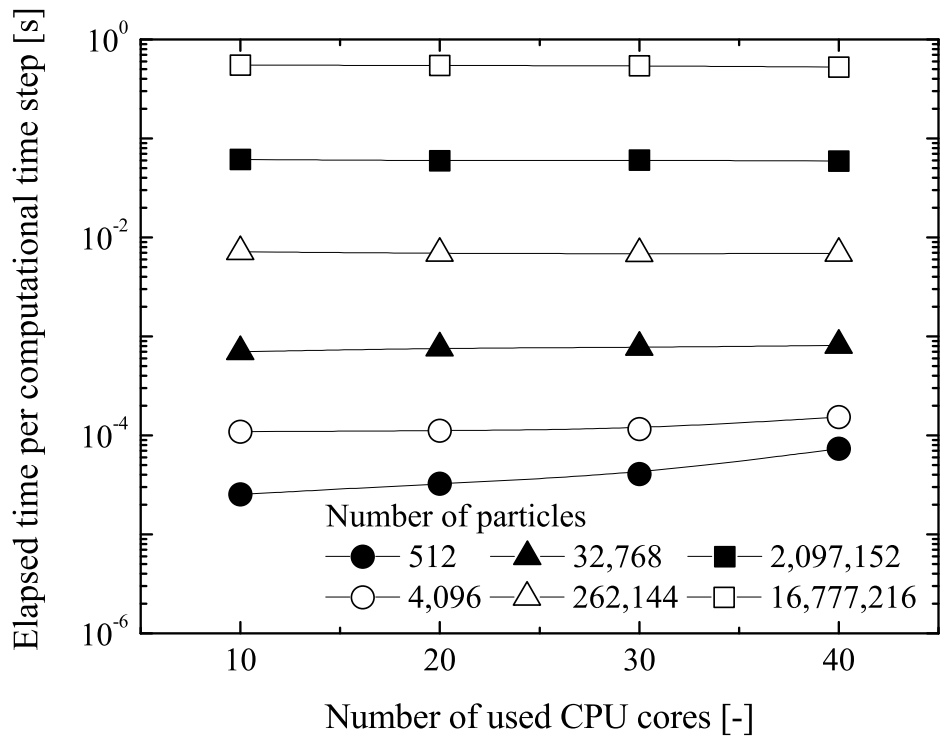

(a) Creating the list of particle pairs in contact candidate

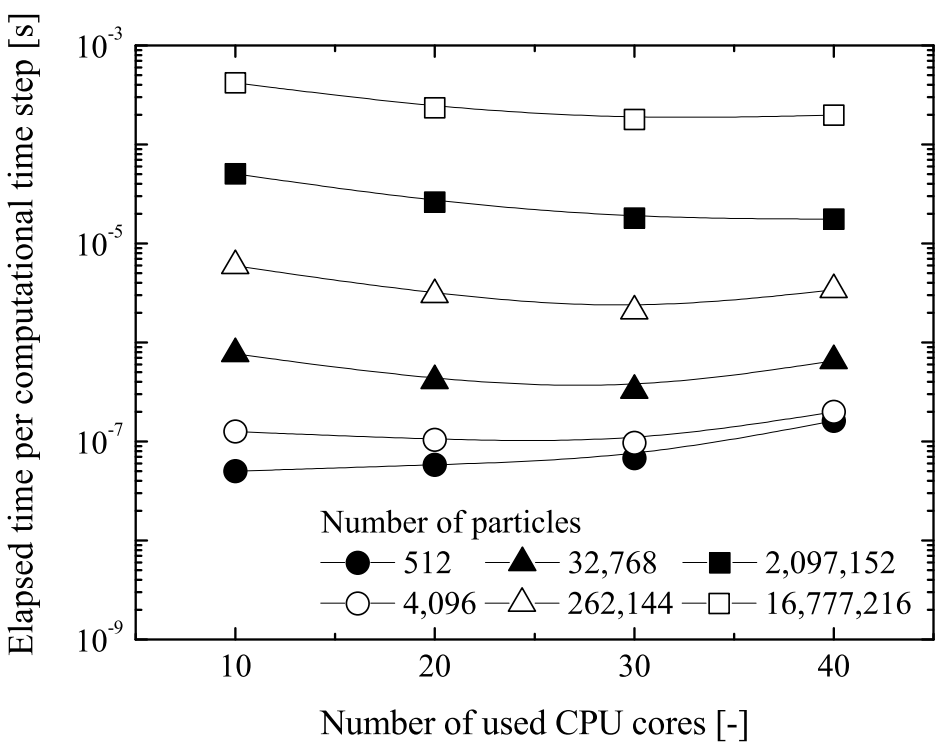

(b) Calculation of contact force

Figure 6: Parallel performance of ppohDEM on shared memory. 


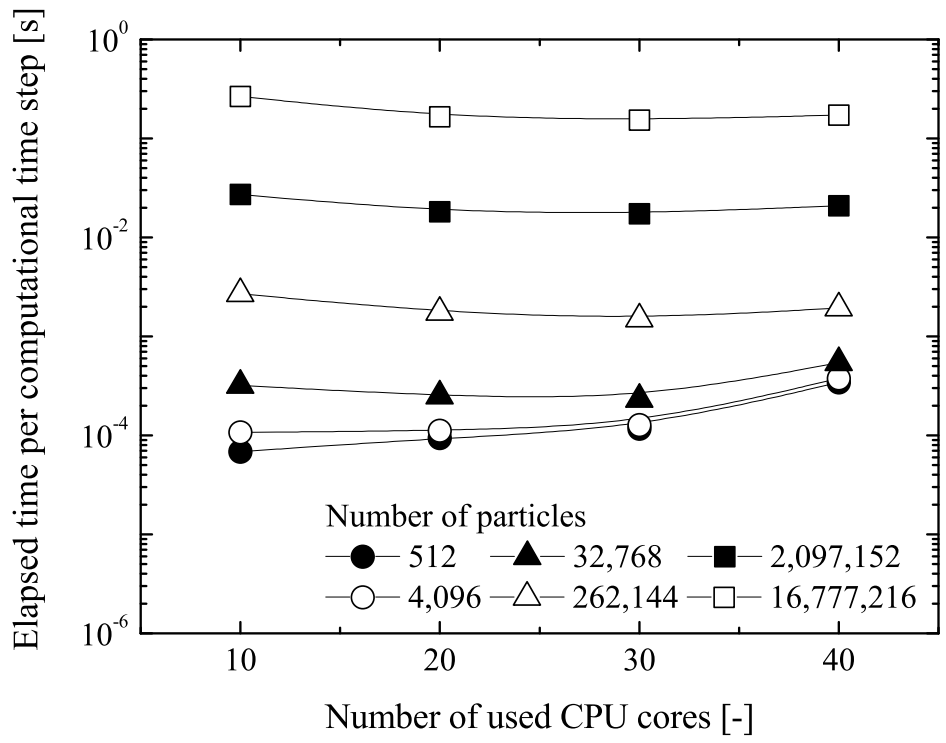

(a) Creating the list of particle pairs in contact candidate

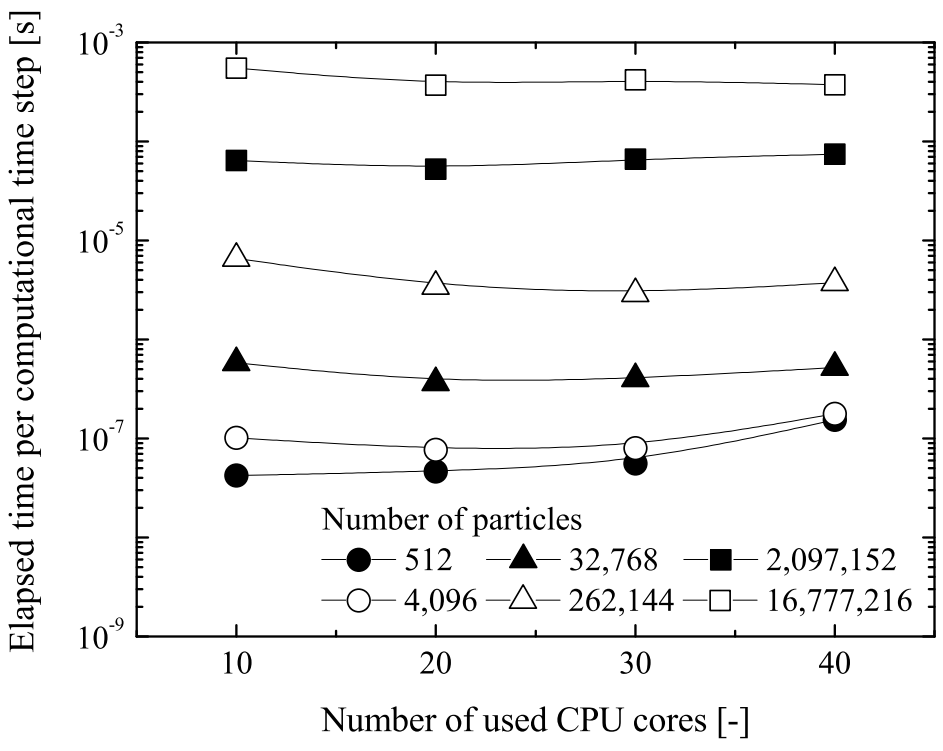

(b) Calculation of contact force

Figure 7: Parallel performance of DEMIGLACE on shared memory. 
scaling test, with the computational region uniformly decomposed by the number of MPI processes. Here, the MPI process is distributed among CPU units, and the 10 cores in a CPU are parallelized on shared memory using 10 threads with OpenMP. For ppohDEM, the computational times for the creation of contact candidate pairs and the calculation of contact force linearly decreased as the number of MPI processes increased. A similar result for the creation of contact candidate was found for DEMIGLACE. However, the parallel performance for the calculation of contact force was not better than that of ppohDEM because MPI communication data in DEMIGLACE is larger than ppohDEM due to summing the contact force using a reference table. It should be mentioned that the parallel performance of OpenDEM_C (using no sort technique) was slightly lower than that of ppohDEM due to the cost of packing/unpacking operations for MPI communication data. Thus, we conclude that the cost and complexity of packing/unpacking operations for MPI communication data do not exist in ppohDEM and DEMIGLACE, so even though the benefits of the sort technique were not evident under the strong scaling test, the simplicity of MPI communication argues strongly in favor of ppohDEM and DEMIGLACE.

Next, in order to investigate the optimum balance between the number of MPI processes and the number of OpenMP threads, we measured the computational time for different numbers of MPI processes and OpenMP threads. For a fixed number of CPU cores (40), the relationship between the number of MPI processes $N_{\text {proc }}$ and the number of OpenMP threads $N_{\text {thread }}$ is given by

$$
N_{\text {thread }}=40 / N_{\text {proc }}
$$

All other test conditions were as used in the measurement of Fig. 8. Our results are shown in Fig. 9. The computational performance of ppohDEM was improved by increasing the number of MPI processes and decreasing the number of OpenMP threads. Especially for the creation of contact candidate pairs, the performance of ppohDEM nears that of DEMIGLACE at 8 MPI processes. This indicates that reducing the number of threads per MPI process improves cache sharing and hides the low performance of shared memory parallelization, and that the parallel performance of ppohDEM will be maximized when the number of threads is minimized. 


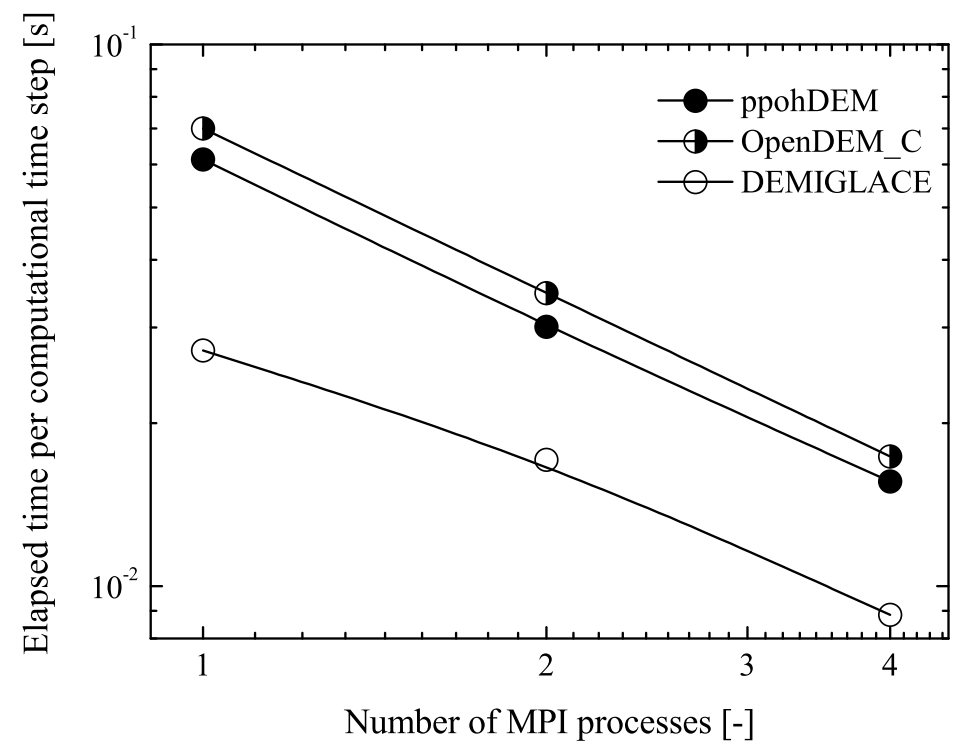

(a) Creating the list of particle pairs in contact candidate

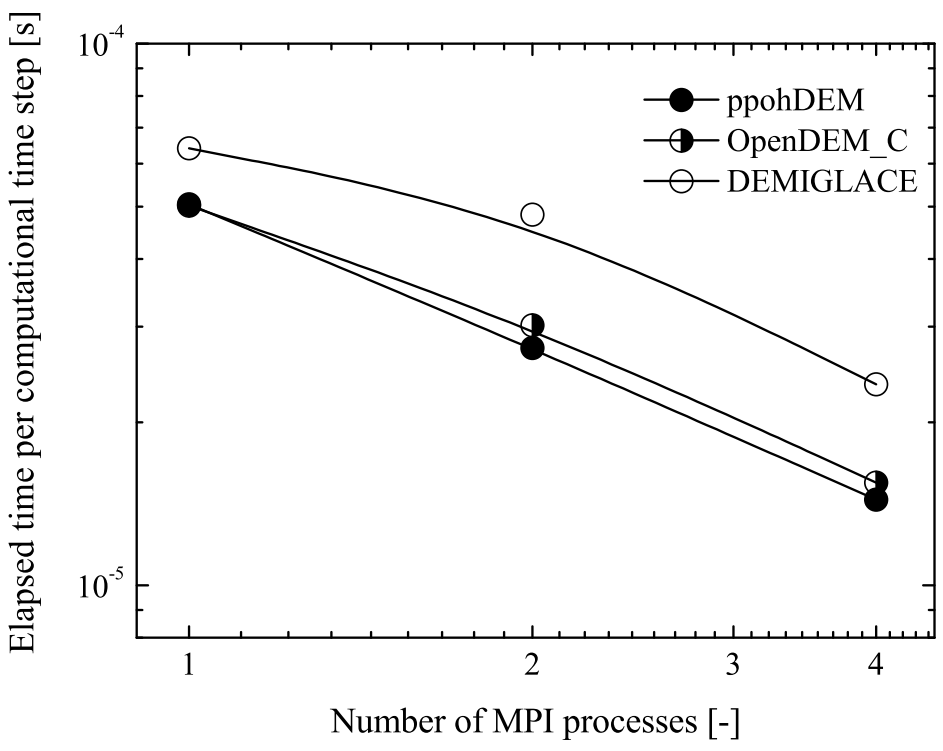

(b) Calculation of contact force

Figure 8: Comparison of ppohDEM and DEMIGLACE parallel performance on distributed memory 


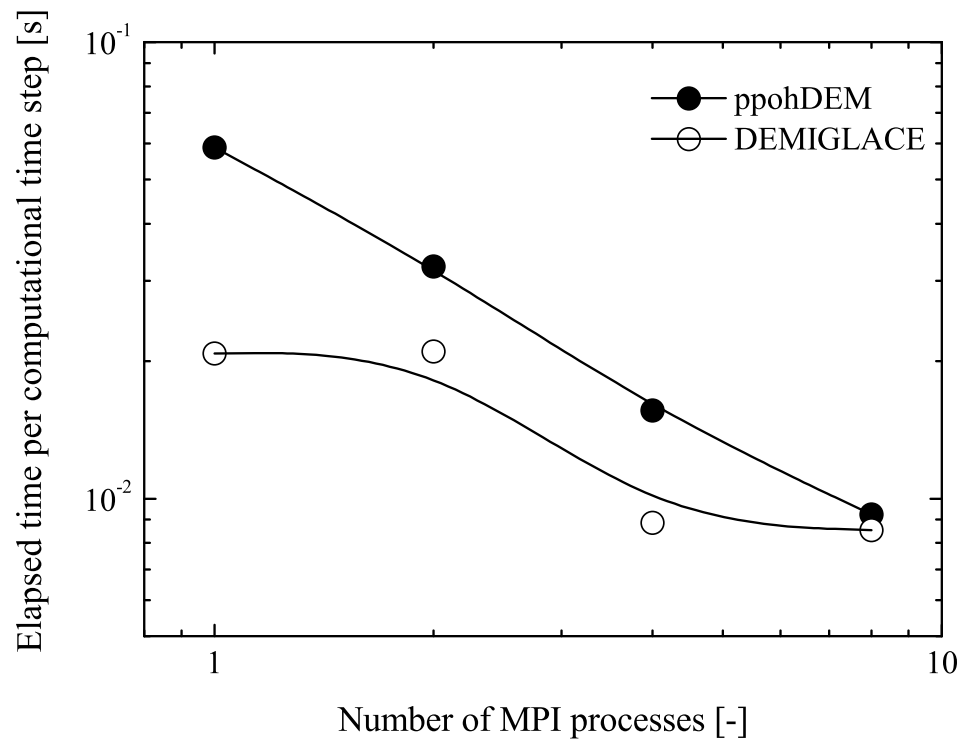

(a) Creating the list of particle pairs in contact candidate

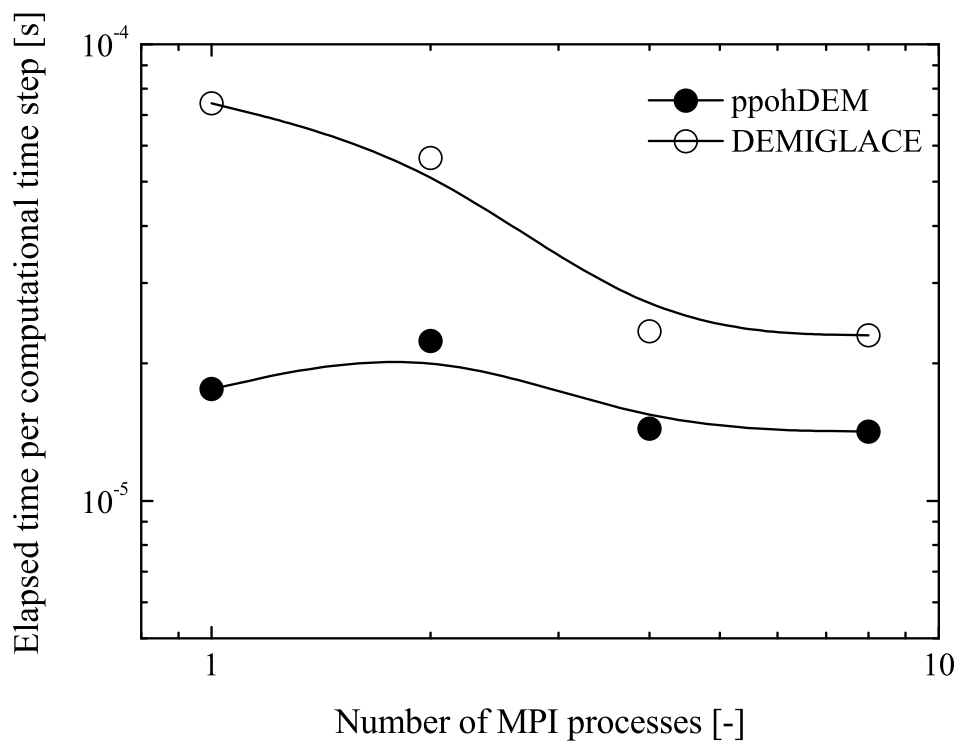

(b) Calculation of contact force

Figure 9: Effects of the number of MPI processes and the number of OpenMP threads on computational performances of ppohDEM and DEMIGLACE, when total usage of CPU cores is fixed at 40 . 


\section{Conclusion}

The open source code for parallelized DEM (ppohDEM) was developed using OpenMP and MPI, and included a new search algorithm for candidate contact pairs of particles (DSM). DSM reduced elapsed search time by 20 $\%$. The performance benefits of DSM were also confirmed, as were the benefits its sequential memory communication for parallelization on distributed memory. With performance approaching that of DEMIGLACE, we believe that ppohDEM is better suited to distributed memory parallel computers with multi-core CPUs than to many-core CPUs.

Overall, ppohDEM succeeds in bringing the benefits of parallel computing to a broader user base, by hiding the complexity of fundamental componentry. We hope that ppohDEM can help scientists and engineers develop more parallelized code and reap the benefits of parallel computing in the application of DEM.

\section{Acknowledgement}

This work is supported by Japan Science and Technology Agency (JST) under the Core Research of Evolutional Science and Technology (CREST) project "ppOpen-HPC: Open Source Infrastructure for Development and Execution of Large-Scale Scientific Applications on Post-Peta-Scale Supercomputers with Automatic Tuning."

\section{Appendix A. Details of Source Codes used for Comparison with ppohDEM in Performance Testing}

OpenDEM_A includes the fundamental frameworks of ordinary DEM code. OpenDEM_B applies the action-reaction law to the summation of contact force given in OpenDEM_A. OpenDEM_C implements the descend storage method (DSM) in OpenDEM_B. Finally, based on OpenDEM_C, we integrated our sort technique to create ppohDEM.

In OpenDEM_A, methods for storing particle labels in cells and searching neighbor cells to create particle pair lists are given in Code A.1 and Code A.2, respectively. In Sub_Cell of Code A.1, the cell data array Norderp is initialized, after which the number of particles in each cell, Numcl, is counted and those particle labels are stored in the Norderp array in ascending order by particle label. Next, in Sub_List of Code A.2, for each particle $i$, contact 


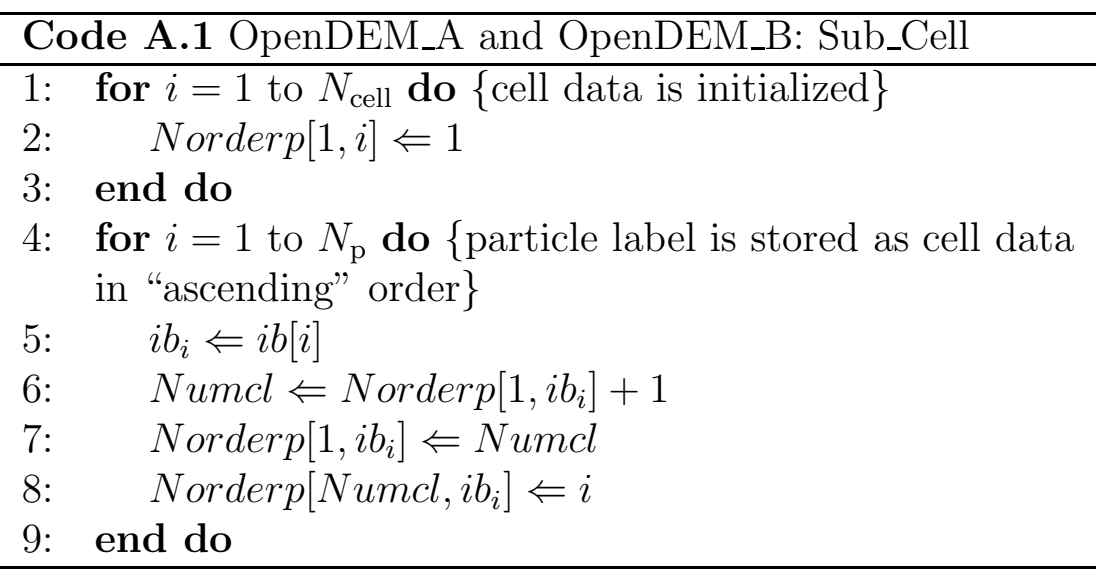

candidate pair particles $j$ are searched in the neighboring 27 cells based on the cell data in Norderp. The difference between this approach and the approach used in ppohDEM is that, in this approach, each particle makes pairs with all contact candidate particles. This is because the action-reaction law is not used to sum up inter-particle contact forces in OpenDEM_A. Thus, the number of particle pairs produced by this code is twice that of the others.

In contrast, OpenDEM_B and the OpenDEM_C use the action-reaction law to sum up inter-particle contact forces for each particle, so the if statement at line 10 in Code A.2 is changed to "if $j$ is larger than $i$ then" in the Sub_List for OpenDEM_B.

OpenDEM_C also integrates DSM into Sub_Cell. Particle labels are not sorted by cell label; the code for Sub_Cell in OpenDEM_C is identical to that for ppohDEM, excepting the quicksort routine. For Sub_List of OpenDEM_C, DSM lets us eliminate the if statement at line 10 in Code A.2, making the code identical to that of ppohDEM except for the do loop region for searching neighbor cells.

\section{Appendix B. Raw data for parallel performance}

Raw computation times for ppohDEM and DEMIGLACE are listed in Tables B.1 and B.2. These times are also graphed in Figs. 6 and 7, respectively. 


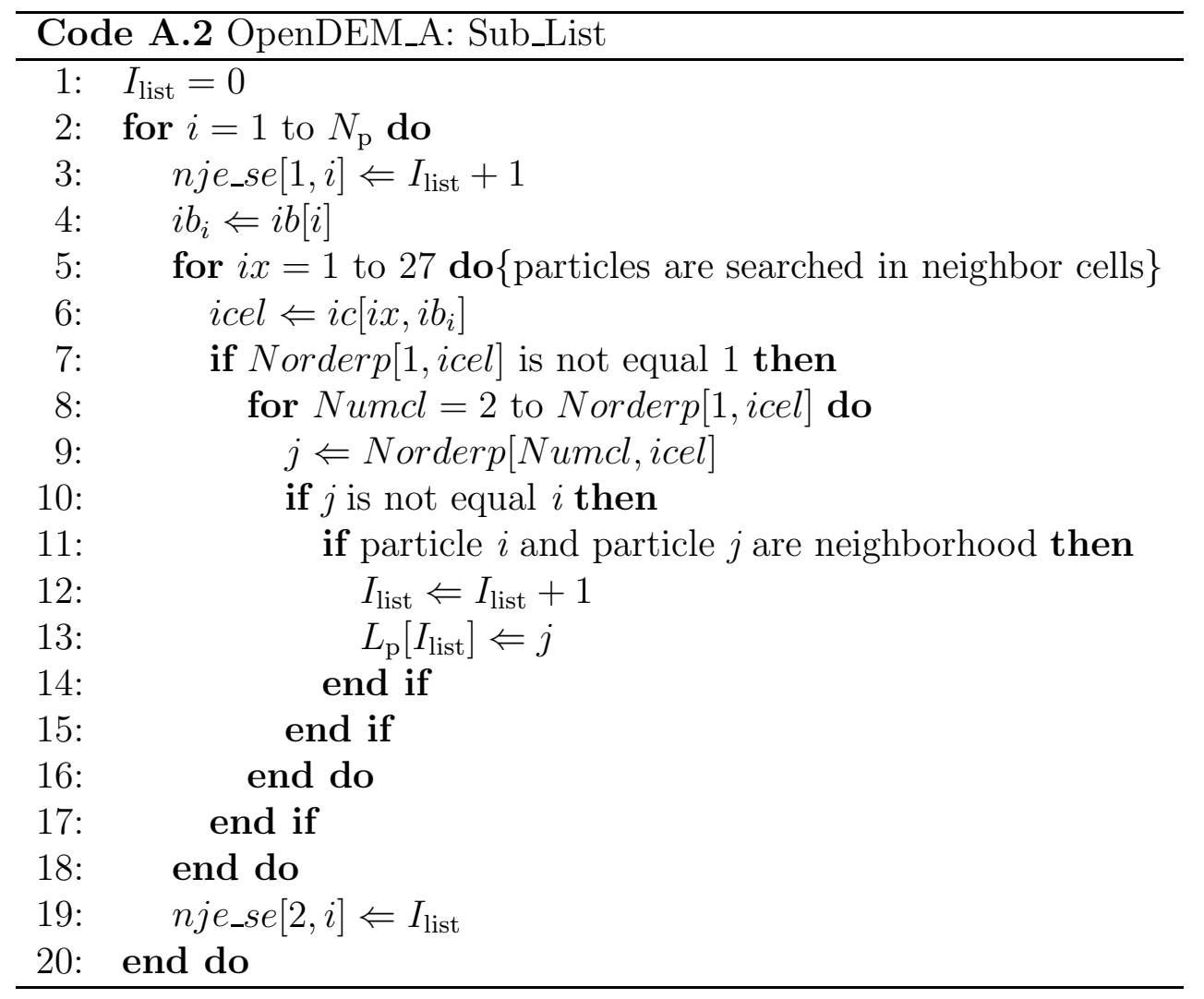

\section{References}

[1] CREST project, http://www.jst.go.jp/kisoken/crest/en/ research_area/ongoing/areah22-2.html (2010).

[2] A. F. Oskooi, D. Roundy, M. Ibanescu, P. Bermel, J. D. Joannopoulos, S. G. Johnson, MEEP: A flexible free-software package for electromagnetic simulations by the FDTD method, Computer Physics Communications 181 (3) (2010) 687-702.

[3] M. Valiev, E. J. Bylaska, N. Govind, K. Kowalski, T. P. Straatsma, H. J. Van Dam, D. Wang, J. Nieplocha, E. Apra, T. L. Windus, et al., Nwchem: a comprehensive and scalable open-source solution for large scale molecular simulations, Computer Physics Communications 181 (9) (2010) 1477-1489. 
Table B.1: Raw data for parallel performance under ppohDEM

\begin{tabular}{c|r|r|r|r|r|r}
\hline & \multicolumn{6}{|c}{ Number of particles } \\
\cline { 2 - 7 } & 512 & 4096 & 32768 & 262144 & 2097152 & 16777216 \\
\hline $\begin{array}{c}\text { Number of } \\
\text { CPU cores }\end{array}$ & \multicolumn{7}{|c}{ Elapsed time of contact force calculation [ms] } \\
\hline 10 & 0.00005027 & 0.00012574 & 0.00076532 & 0.00594577 & 0.05050489 & 0.42070886 \\
\hline 20 & 0.00005801 & 0.00010383 & 0.00041106 & 0.00302067 & 0.02611666 & 0.23426860 \\
\hline 30 & 0.00006777 & 0.00009672 & 0.00032633 & 0.00207816 & 0.01809109 & 0.17858897 \\
\hline 40 & 0.00016147 & 0.00019960 & 0.00065328 & 0.00341336 & 0.01759215 & 0.19727874 \\
\hline $\begin{array}{c}\text { Number of } \\
\text { CPU cores }\end{array}$ & \multicolumn{7}{|c}{ Elapsed time of contact candidate pair list creation [ms] } \\
\hline 10 & 0.02539301 & 0.10866225 & 0.70053244 & 7.19017029 & 61.35348415 & 551.44903541 \\
\hline 20 & 0.03245497 & 0.11122286 & 0.76130331 & 6.89072728 & 59.38927571 & 545.15575409 \\
\hline 30 & 0.04055834 & 0.11625051 & 0.77346206 & 6.85892522 & 60.58066177 & 540.99229813 \\
\hline 40 & 0.07321930 & 0.15266716 & 0.80942988 & 6.90003951 & 58.81659428 & 524.63596821 \\
\hline
\end{tabular}

[4] ppOpen-HPC project, http://ppopenhpc.cc.u-tokyo.ac.jp/ wordpress/ (2013).

[5] P. A. Cundall, O. D. L. Strack, A discrete numerical model for granular assemblies, Geotechnique 29 (1) (1979) 47-65.

[6] J. Kano, F. Saito, Correlation of powder characteristics of talc during planetary ball milling with the impact energy of the balls simulated by the particle element method, Powder Technology 98 (2) (1998) 166-170.

[7] E. Tijskens, H. Ramon, J. Baerdemaeker, Discrete element modelling for process simulation in agriculture, Journal of Sound and Vibration 266 (3) (2003) 493-514.

[8] U. Tüzün, J. Baxter, D. Heyes, Analysis of the evolution of granular stress-strain and voidage states based on dem simulations, Philosophical Transactions A 362 (1822) (2004) 1931-1951.

[9] H. Zhu, Z. Zhou, R. Yang, A. Yu, Discrete particle simulation of particulate systems: A review of major applications and findings, Chemical Engineering Science 63 (23) (2008) 5728-5770.

[10] P. Cleary, Industrial particle flow modelling using discrete element 
Table B.2: Raw data for parallel performance under DEMIGLACE

\begin{tabular}{c|r|r|r|r|r|r}
\hline & \multicolumn{6}{|c}{ Number of particles } \\
\cline { 2 - 7 } & 512 & 4096 & 32768 & 262144 & 2097152 & 16777216 \\
\hline $\begin{array}{c}\text { Number of } \\
\text { CPU cores }\end{array}$ & \multicolumn{7}{|c}{ Elapsed time of contact force calculation [ms] } \\
\hline 10 & 0.00004236 & 0.00010154 & 0.00058325 & 0.00657241 & 0.06405114 & 0.55067090 \\
\hline 20 & 0.00004639 & 0.00007671 & 0.00036382 & 0.00344163 & 0.05234543 & 0.37026421 \\
\hline 30 & 0.00005602 & 0.00008014 & 0.00040131 & 0.00288653 & 0.06634877 & 0.42111251 \\
\hline 40 & 0.00015419 & 0.00017757 & 0.00052082 & 0.00373730 & 0.07425072 & 0.37433777 \\
\hline $\begin{array}{c}\text { Number of } \\
\text { CPU cores }\end{array}$ & \multicolumn{7}{|c|}{ Elapsed time of contact candidate pair list creation [ms] } \\
\hline 10 & 0.06814957 & 0.10726213 & 0.32046616 & 2.68740833 & 27.23715353 & 264.78058636 \\
\hline 20 & 0.09365177 & 0.11134267 & 0.25031745 & 1.74586475 & 18.10691833 & 165.37939072 \\
\hline 30 & 0.11645842 & 0.12723148 & 0.23067057 & 1.49775505 & 17.31696665 & 153.71441603 \\
\hline 40 & 0.34247220 & 0.37729442 & 0.53991795 & 1.93348567 & 20.81163009 & 172.20116854 \\
\hline
\end{tabular}

method, Engineering Computations: Int. J. for Computer-Aided Engineering 26 (6) (2009) 698-743.

[11] D. Nishiura, Y. Wakita, A. Shimosaka, Y. Shirakawa, J. Hidaka, Estimation of power during dispersion in stirred media mill by dem-les simulation, Journal of Chemical Engineering of Japan 43 (10) (2010) 841-849.

[12] Large-scale atomic/molecular massively parallel simulator, LAMMPS, http://lammps.sandia.gov. (2004).

[13] ESyS-Particle: HPC discrete element modelling software, https:// launchpad.net/esys-particle (2004).

[14] J. Kozicki, F. Donzé, Yade-open dem: an open-source software using a discrete element method to simulate granular material, Engineering Computations 26 (7) (2009) 786-805.

[15] OpenFOAM v2.0.0: New lagrangian functionality, http://www . openfoam.org/version2.0.0/lagrangian.php (2004).

[16] D. Nishiura, H. Sakaguchi, Parallel-vector algorithms for particle simulations on shared-memory multiprocessors, Journal of Computational Physics 230 (5) (2011) 1923-1938. 
[17] D. Rapaport, Multi-million particle molecular dynamics, I. design considerations for vector processing, Computer Physics Communications $62(2-3)$ (1991) 198-216.

[18] H. Sakaguchi, D. Nishiura, DEMIGLACE, http://www. jamstec.go . $\mathrm{jp} / \mathrm{j} /$ about/patent/innovation/vol11.pdf (2009).

[19] M. Sawley, P. Cleary, A parallel discrete element method for industrial granular flow simulations, EPFL Supercomputing Review 11 (1999) 239.

[20] A. Maknickas, A. Kačeniauskas, R. Kačianauskas, R. Balevičius, A. Džiugys, Parallel DEM software for simulation of granular media, Informatica 17 (2) (2006) 207-224.

[21] W. Chang, S. Hsieh, Parallelization of discrete element simulation, Journal of the Chinese Institute of Engineers 32 (6) (2009) 825-841.

[22] J. Walther, I. Sbalzarini, Large-scale parallel discrete element simulations of granular flow, Engineering Computations: Int. J. for ComputerAided Engineering 26 (6) (2009) 688-697.

[23] D. Rapaport, Multi-million particle molecular dynamics II. design considerations for distributed processing, Computer Physics Communications 62 (1991) 217-228.

[24] S. Plimpton, Fast parallel algorithms for short-range molecular dynamics, Journal of Computational Physics 117 (1) (1995) 1-19.

[25] G. Kohring, Dynamic load balancing for parallelized particle simulations on MIMD computers, Parallel Computing 21 (4) (1995) 683-693.

[26] F. Fleissner, P. Eberhard, Load balanced parallel simulation of particlefluid DEM-SPH systems with moving boundaries, Advances in Parallel Computing 15 (2008) 37-44.

[27] T. Iwai, C. W. Hong, P. Greil, Fast particle pair detection algorithms for particle simulations, International Journal of Modern Physics C 10 (05) (1999) 823-837. 